\title{
Cobalamin-dependent methionine synthase: the structure of a methylcobalamin-binding fragment and implications for other $B_{12}$-dependent enzymes
}

\section{Catherine Luschinsky Drennan, Rowena G Matthews and Martha L Ludwig}

\author{
University of Michigan, Ann Arbor, USA
}

\begin{abstract}
Cobalamin-dependent methionine synthase is a large enzyme composed of structurally and functionally distinct regions. Recent studies have begun to define the roles of several regions of the protein. In particular, the structure of a $27 \mathrm{kDa}$ cobalamin-binding fragment of the enzyme from Escherichia coli has been determined by X-ray crystallography, and has revealed the motifs and interactions responsible for recognition of the cofactor. The amino acid sequences of several adenosylcobalamin-dependent enzymes, the methylmalonyl coenzyme A mutases and glutamate mutases, show homology with the cobalamin-binding region of methionine synthase and retain conserved residues that are determinants for the binding of the prosthetic group, suggesting that these mutases and methionine synthase share common three-dimensional structures.
\end{abstract}

Current Opinion in Structural Biology 1994, 4:919-929

\section{Introduction}

Cobalamin-dependent methionine synthases are key enzymes in the one-carbon metabolism of mammals and microorganisms. Impairment of enzyme activity, either as a result of cobalamin $\left(\mathrm{B}_{12}\right)$ deficiency $[1,2]$ or following exposure to nitrous oxide [3-6], results in megaloblastic anemia and spinal cord degeneration in humans.

The best characterized methionine synthase is the metH gene product from Escherichia coli, which has been overexpressed and purified to homogeneity [7]. The enzyme uses methylcobalamin as an intermediate in the transfer of a methyl group from methyltetrahydrofolate $\left(\mathrm{CH}_{3}-\right.$ $\mathrm{H}_{4}$ folate) to homocysteine, forming $\mathrm{H}_{4}$ folate and methionine. The cobalamin is alternately demethylated by homocysteine and methylated by $\mathrm{CH}_{3}-\mathrm{H}_{4}$ folate:

$$
\begin{aligned}
& \text { E-methylcobalamin }+ \text { homocysteine } \rightarrow \\
& \text { E.cob(I)alamin }+ \text { methionine }
\end{aligned}
$$

A diagram of the catalytic cycle is shown in Fig. 1. In methylcobalamin, the methyl group is directly bonded to the cobalt of cobalamin, and during the catalytic cycle this carbon-cobalt bond is broken and then reformed. As implied by equations 1 and 2, the carbon-cobalt bond is formally cleaved heterolytically, with transfer of a methyl carbocation. The enzyme-bound cob(I)alamin species has been shown to be a kinetically competent intermediate in catalysis [8]. The free cob(I)alamin species is highly reactive, and serves both as an excellent nucleophile [9], and as a strong reductant [10].

The reactive $\operatorname{cob}(\mathrm{I})$ alamin intermediate occasionally undergoes oxidation, to form an inactive cob(II)alamin enzyme species. In vitro, this oxidation occurs once in every 100-2000 turnovers, depending on the degree of anaerobiosis achieved during turnover, and on the constituents of the assay reaction $\left[11,12^{\circ}\right]$ Reactivation of the cob(II)alamin enzyme requires a reductive methylation to form methylcobalamin enzyme; S-adenosylmethionine (AdoMet) serves as the methyl donor, and the physiological electron donor is believed to be flavodoxin $[11,13,14]$. AdoMet is required in catalytic amounts and is not a methyl donor in primary turnover (Fig. 1).

Methionine synthase thus catalyzes three different methyl transfers to or from the prosthetic group. $\mathrm{S}_{\mathrm{N}} 2$ mechanisms, which have been assumed for the primary reaction cycle, impose strong geometric requirements on the reactants. How then are the substrates homocysteine and $\mathrm{CH}_{3}-\mathrm{H}_{4}$ folate and the activator AdoMet alternately positioned for methyl transfer? Recent X-ray analysis emphasizes the difficulty of access to the cobalt and its methyl ligand. The structure of the cobalamin-binding domains, described below, shows protein residues capping the top face of the corrin, protecting the methyl 


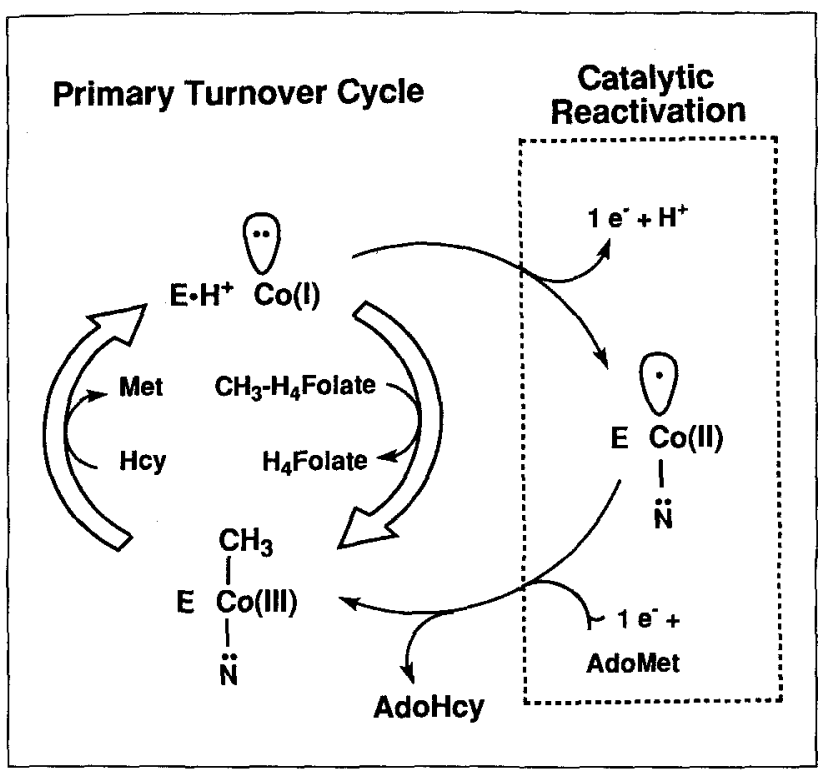

Fig. 1. The catalytic cycle of methionine synthase. During turnover the cobalamin prosthetic group is alternately methylated with a methyl group from $\mathrm{N}^{5}$-methyltetrahydrofolate, and demethylated with transfer of the methyl group to homocysteine (Hcy). Demethylation results in the formation of enzyme-bound cob(I)alamin which is assumed to be a four-coordinate species. The electrons remain in the $d_{z}{ }^{2}$ orbital of cob(1)alamin, which is perpendicular to the plane of the corrin macrocycle. In contrast, the methylated cofactor is six-coordinate, with a nitrogen ligand from His759 occupying the lower axial coordination position [42 $\left.{ }^{\bullet}\right]$. Enzyme demethylation is depicted as resulting in protonation of a group on the enzyme, and we postulate that the His759-Asp757 pair accepts the proton. The cob(l)alamin prosthetic group is occasionally oxidized during turnover, resulting in the formation of inactive cob(II)alamin. One-electron oxidation of the cofactor is accompanied by proton release [38*]. Return of enzyme to the catalytic cycle requires a reductive methylation of the cofactor, in which the methyl group is provided by S-adenosylmethionine (AdoMet) rather than by $\mathrm{CH}_{3}-$ $\mathrm{H}_{4}$ folate. In Escherichia coli, reduced flavodoxin is thought to be the electron donor [11].

group. How the enzyme regulates access to the upper face of the corrin remains an interesting but unresolved issue.

The mammalian enzymes have many properties in common with the better-studied methionine synthase from $E$. coli, suggesting that in many respects the latter enzyme may serve as a model for the mammalian proteins. None of the mammalian genes coding for methionine synthase enzymes has yet been cloned and sequenced, although several mammalian enzymes have been partially purified [15-17]. The porcine enzyme exhibits the same activity per nanomole of cobalamin as the enzyme from $E$. coli [15]; as with the bacterial enzyme, activity is dependent on the presence of AdoMet and a reducing system [18]. Both the mammalian enzyme [18] and the enzyme from E. coli $[19,20]$ form methylcobalamin following incubation with AdoMet and a reducing system.

The $\mathrm{B}_{12}$-dependent enzymes that contain adenosylcobalamin rather than methylcobalamin do not form cob(I)alamin during the catalytic cycle, but rather catalyze a homolytic cleavage of the carbon-cobalt bond of adenosylcobalamin to form a 5'-deoxyadenosyl radical and cob(II)alamin. Methylmalonyl coenzyme A (CoA) mutase, the second of the two $B_{12}$-dependent enzymes found in humans, is an adenosylcobalamin-dependent enzyme [21], as are a number of bacterial amino acid mutases [22,23], ethanolamine ammonia-lyase [24], and ribonucleotide reductase from Lactobacillus leichmanii [25]. In these enzymes, the $5^{\prime}$-deoxyadenosyl radical appears to play the role of a radical initiator, abstracting a hydrogen atom either directly from the substrate or from an amino acid residue on the protein. Information from structures and sequences, reported during 1993-1994, has begun to address the resemblances and contrasts between the methyl- and adenosylcobalamin classes of $\mathrm{B}_{12}$-dependent enzymes. A central but open question for enzymologists is how the cobalamin cofactor is influenced by the protein to favor heterolytic cleavage of the carbon-cobalt bond in methionine synthase and homolytic cleavage of the carbon-cobalt bond in adenosylcobalamin-dependent enzymes.

\section{Dissection of cobalamin-dependent methionine synthase into functional and structural units}

Cobalamin-dependent methionine synthase is one of the largest polypeptides in E. coli, with 1227 residues and a molecular weight of 136087 . To resolve ambiguities in the DNA sequence analyses $[7,26]$, the sequence of the carboxy-terminal portion of the protein was determined from the sizes of peptide fragments using electrospray mass spectrometry [27 $\left.{ }^{\circ}\right]$. Initial examination of the amino acid sequence of methionine synthase [7] failed to detect significant homology with any other protein sequences. The starting strategy for defining functional and structural regions of the protein was limited proteolysis of the native holoenzyme. Digestion with trypsin initially cleaves the protein at Arg896 to produce two fragments of 98 and $37 \mathrm{kDa}$, and amino-terminal sequencing established that the smaller fragment was derived from the carboxyl terminus of the protein. As judged by the red color of the amino-terminal $98 \mathrm{kDa}$ piece, cobalamin remained bound to this fragment. A smaller $28 \mathrm{kDa}$ fragment derived by further digestion with trypsin, consisting of residues 644-896 of the holoenzyme, retained the bound cobalamin. A related fragment extending from residue 651 to residue 896 was generated in crystallization experiments [28]. The $28 \mathrm{kDa}$ fragment stabilized bound $\operatorname{cob}(\mathrm{II})$ alamin against oxidation and protected the bound methylcobalamin against photolysis, properties that distinguish enzyme-bound from free cobalamin derivatives. However, the fragment was unable to catalyze demethylation of the methylcobalamin cofactor by homocysteine or methylation of $\mathrm{cob}(\mathrm{I})$ alamin by $\mathrm{CH}_{3^{-}}$ $\mathrm{H}_{4}$ folate $\left[12^{\bullet}\right]$.

The $98 \mathrm{kDa}$ fragment, extending from residues 1 to 896 and isolated from tryptic digests of holoenzyme containing methylcobalamin, is initially fully active in catalyzing methyl transfer from $\mathrm{CH}_{3}-\mathrm{H}_{4}$ folate to homocysteine [12॰]. Thus this fragment must retain the residues necessary for binding both homocysteine and $\mathrm{CH}_{3} \mathrm{H}_{4}$ folate, 
as well as residues necessary for catalysis of methyl transfer to and from the enzyme-bound cobalamin. However, the enzyme activity gradually diminishes during turnover and the cob(II)alamin enzyme can no longer be reactivated by reductive methylation with AdoMet. These observations suggest that the carboxy-terminal fragment is essential for reductive activation.

The carboxy-terminal $37 \mathrm{kDa}$ unit carries the binding site for the activator, AdoMet. After intact protein has been irradiated with ultraviolet light in the presence of tritiated AdoMet, the label is associated with this fragment. The isolated fragment can also be labeled with AdoMet [12॰]. However, the mechanism by which methyl transfer from AdoMet is coupled to reduction of cob(II)alamin (Fig. 1) remains to be elucidated. Several other enzymes are also activated by reactions involving flavodoxin and AdoMet: pyruvate formate-lyase [29,30], anaerobic ribonucleotide reductase $\left[31,32^{\circ}\right]$ and, possibly, biotin synthase $\left[33,34^{\circ}\right]$. Activation of pyruvate formate-lyase results in the formation of an organic radical, localized to the $\alpha$-carbon of a specific glycine residue [ $\left.35^{\circ}\right]$, and a glycine radical is also thought to be formed in the activation of anaerobic ribonucleotide reductase $\left[36^{\bullet}, 37^{\bullet}\right]$. One-electron reduction of AdoMet and homolytic cleavage of the carbon-sulfur bond yields a 5'-deoxyadenosyl radical which, in turn, can generate the protein radical by hydrogen abstraction [35०]. In the case of methionine synthase, no evidence for a protein radical has been obtained, but one-electron reduction of AdoMet to form $S$-adenosylhomocysteine (AdoHcy) and a methyl radical is a possible pathway leading to formation of methylcobalamin.

Experiments to elucidate the mode of inactivation of cobalamin-dependent methionine synthase by the anaesthetic gas nitrous oxide provide evidence for close approach of the carboxy-terminal domain and the cobalamin cofactor $\left[38^{\bullet}, 39^{\bullet}\right]$. In an electrochemical cell under nitrous oxide $\left(\mathrm{N}_{2} \mathrm{O}\right)$ the enzyme catalyzes the one electron reduction of this gas according to equation 3 [38*]:

$$
\begin{aligned}
& \mathrm{EH}^{+} \cdot \operatorname{cob}(\mathrm{I}) \text { alamin }+ \mathrm{N}_{2} \mathrm{O} \rightarrow \\
& \mathrm{E} \cdot \operatorname{cob}(\mathrm{II}) \text { alamin }+\mathrm{N}_{2}+\mathrm{OH} \cdot
\end{aligned}
$$

The hydroxyl radical is presumably generated from the oxygen of nitrous oxide as this gas reacts with cobalamin bound to the $28 \mathrm{kDa}$ fragment. This radical is detected because its predominant reaction is abstraction of a hydrogen atom from Val1177 in the carboxy-terminal portion of the protein, to form a valine radical that condenses with the reduced triquat dye used to mediate electron transfer to the enzyme [39॰]. Since the hydroxyl radical is expected to have an extremely short lifetime, it is likely that Val1177, 50 residues from the extreme carboxyl terminus of the protein, lies close in space to the site where nitrous oxide is reduced by $\operatorname{cob}(\mathrm{I})$ alamin.

Recent sequence determinations have helped to define conserved segments of methionine synthase. Two open reading frames with homologies to the $98 \mathrm{kDa}$ amino- terminal domain and the $37 \mathrm{kDa}$ carboxy-terminal domain of MetH have been identified in Mycobacterium leprae [40]. Taken together, the two $M$. leprae sequences show $27 \%$ identity with the full-length MetH sequence of $E$. coli. As shown by the alignment in Fig. 2, the similarity of these two methionine synthase sequences is most evident in the $28 \mathrm{kDa}$ cobalamin-binding portion of the E. coli enzyme, but other strongly conserved regions, including sequences upstream from the cobalamin-binding region, are apparent from the alignment. The roles of the amino-terminal sequences of methionine synthase remain to be determined. At present there is no direct evidence that identifies the substrate-binding sites in methionine synthase. However, alignment of MetH with the sequence for a methyl transferase from Clostridium thermoaceticum $\left[41^{\circ}\right]$ points to a region that may include determinants for the binding of $\mathrm{CH}_{3}-\mathrm{H}_{4}$ folate (Fig. 2). The transferase catalyzes methyl transfer from $\mathrm{CH}_{3}-\mathrm{H}_{4}$ folate to the cobalt center of a corrinoid/iron-sulfur protein, and functions in the reductive acetyl-CoA pathway for $\mathrm{CO}_{2}$ fixation in $C$. thermoaceticum. Thus the reaction catalyzed by the methyl transferase is equivalent to one of the half reactions of methionine synthase (equation 2). The first 200 residues of the deduced methyl transferase sequence align immediately upstream from the cobalamin-binding region of methionine synthase, between residues 356 and 630 , suggesting that this portion of methionine synthase includes the binding site for $\mathrm{CH}_{3}-\mathrm{H}_{4}$ folate and residues necessary to activate this substrate for removal of the methyl group. The notion that methionine synthase from $E$. coli is a modular structure is reinforced by the homology with this methyl transferase. Presumably, the major determinants for binding homocysteine and methionine, neither of which interacts with $C$. thermoaceticum methyl transferase, lie elsewhere in the methionine synthase sequence, perhaps in the amino-terminal 350 residues.

\section{The crystal structure of the methylcobalamin- binding domains of methionine synthase}

The X-ray structure of a $27 \mathrm{kDa}$ cobalamin-binding region of methionine synthase has been determined at a resolution of $3.0 \AA\left[42^{\bullet \bullet}\right]$. This analysis revealed a number of cobalamin-protein interactions that are likely to be characteristic of other cobalamin-dependent proteins.

The fragment that binds methylcobalamin folds into two domains, with the corrin macrocycle embedded in the domain interface (Fig. 3). On binding to methionine synthase, methylcobalamin undergoes a dramatic rearrangement in which the dimethylbenzimidazole nucleotide swings away from the cobalt to open the lower face of the corrin to coordination by a histidine residue from the protein. The 'nucleotide tail' penetrates into the core of the carboxy-terminal $\alpha / \beta$ domain. Thus the recognition of methylcobalamin by the protein involves three sets of protein-cofactor interactions: contacts with the upper face of the corrin, where methylation and demethylation of the prosthetic 


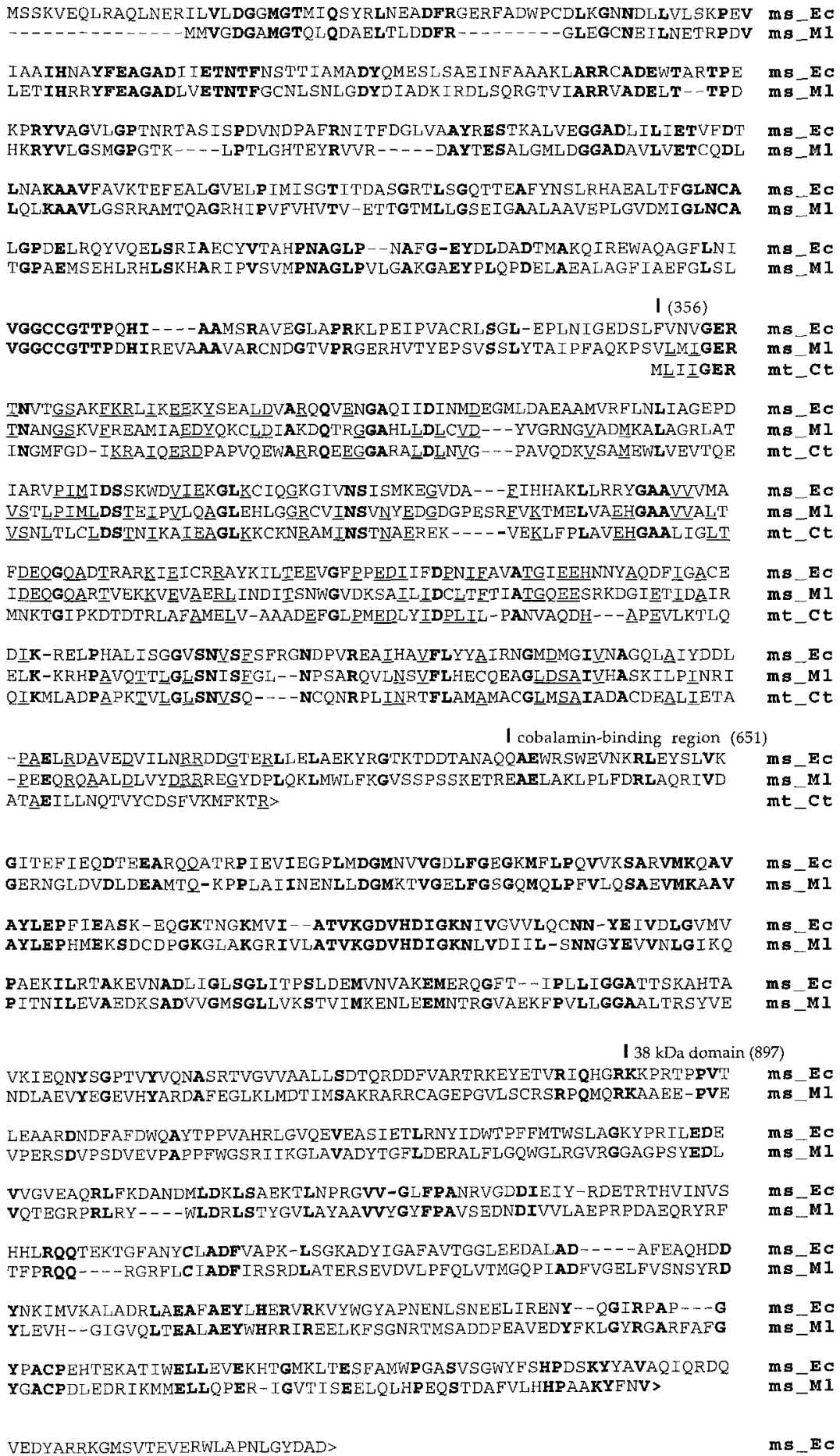

LEAARDNDFAFDWOAYTPPVAHRLGVQEVEASIETLRNY IDWTPF FMTWSLAGKYPRILEDE VPERSDVPSDVEVPAP PFWGSRI I KGLAVADYTGFLDERALFLGQWGLRGVRGGAGPS YEDI

VVGVEAQRLFKDANDMLDKLSAEKTLNPRGVV-GLFRANRVGDDIEIY-RDETRTHVINVS VQTEGRPRLRY - - -WLDRLSTYGVLAYAAVVYGYFPAVSEDNDIVVLAEPRPDAEQRYRF

HHLROOTEKTGF ANYCLADFVAPK-LSGKADY IGAFAVTGGLEEDALAD- - - - AFEAQHDD TFPRQQ- - - RGRFLCIADF IRSRDLATERSEVDVLPFQLVTMGQPIADFVGELFVSNSYRD

YNK IMVKALADRLAEAFAEY $L$ HERVRKVYWGYAPNENLSNEELIRENY- - QGIRPAP - - -G YLEVH - -GIGVOLTEALAEYWHRR IREELKFSGNRTMSADDPEAVEDYFKLGYRGARFAFG

Y PACPEHTEKAT IWELLEVEKHTGMKLTESFAMWPGASVSGWYFSHPDSKYYAVAQIQRDQ YGACPDLEDRI KMMELLQPER-IGVTISEELQLHPEQSTDAFVLHHPAAKYFNV >

ms_Ec ms_MI

ms_EC ms_Ml $\mathbf{m s} \mathbf{E} \mathbf{C}$ $m \mathbf{m} \mathbf{M} 1$ ms_Ec ms MI ms EC $m s 1$ mt_ct ms_Ec ms $M 1$ $m t \_c t$ ms_ec ms_M 1 $m t \_c t$ ms $E C$ ms $M 1$ $m t \_c t$ MB_EC ms_M 1 $m t \_c t$ ms_EC ms Ml $m t \_c t$ ms MI

Fig. 2. Alignment of the deduced amino acid sequence of the metH gene from Escherichia coli $\left[27^{\bullet}\right]$ (ms-Ec) with deduced sequences from two open reading frames from Mycobacterium leprae [40] (ms-MI) that were identified by homology as coding for the 98 and $37 \mathrm{kDa}$ domains of a cobalamin-dependent methionine synthase. Also shown is the alignment of the deduced sequence for a methyltetrahydrofolate-corrinoid/ironsulfur protein methyl transferase from Clostridium thermoaceticum [41 ${ }^{\circ}$ (mt$\mathrm{Ct}$ ). Pairwise identities are underlined in the region that includes the sequence of the methyltransferase and residues conserved in all sequences are shown in bold. Alignments were initially performed with MacVector programs, and were then modified to accord with the structure. group take place; contacts with the dimethylbenzimidazole side chain, which help to anchor the cofactor to the protein; and contacts with the lower face, which allow the protein to modulate the reactivity of the cobalt ion. The amino-terminal domain of the fragment, a fourhelix bundle [43], provides interactions with the upper face of methylcobalamin. In methionine synthase, the upper face contacts are of two types. Hydrophobic side chains such as Val704, Phe708, Leu715 and Leu718 contact the methyl group bonded to cobalt as well as other non-polar substituents of the corrin, while backbone carbonyls hydrogen-bond to the characteristic acetamide side chains of the corrin (Fig. 4). It is expected that this region will differ in enzymes that use adenosyl- 


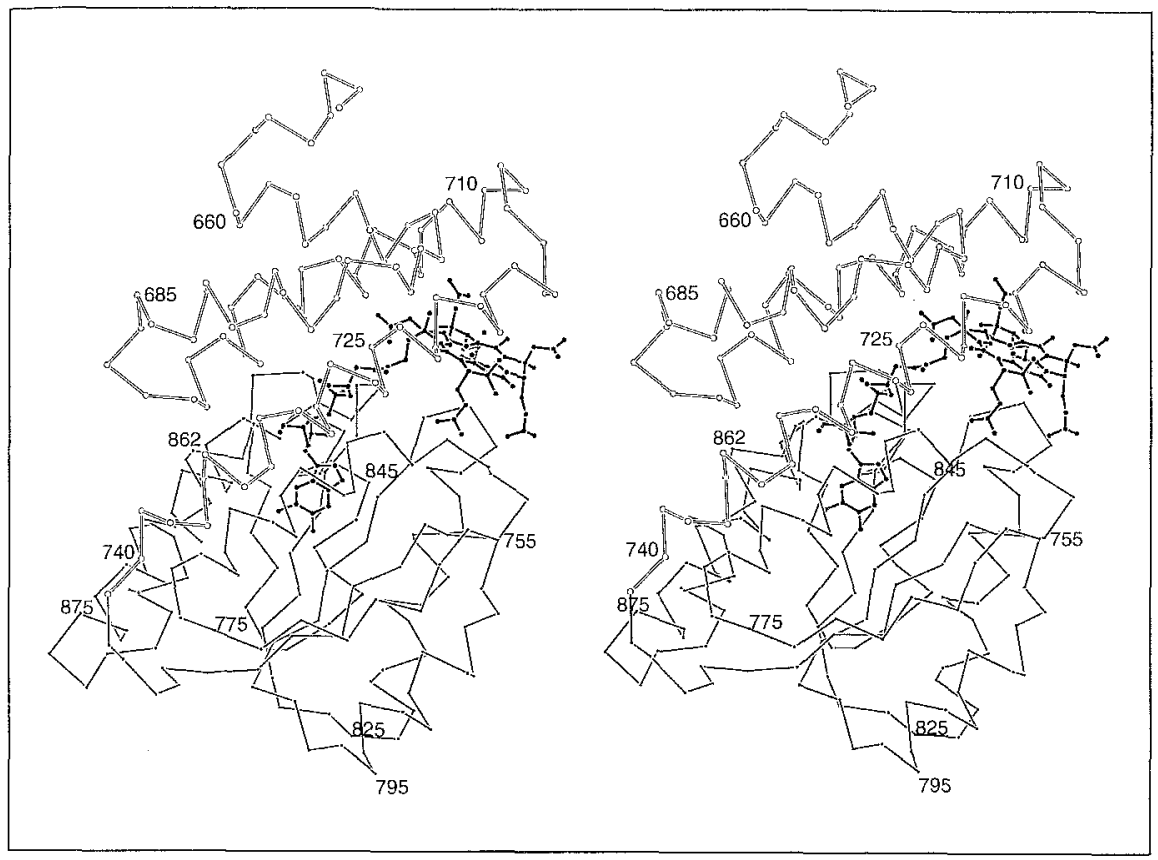

Fig. 3. A stereoview of the fold of the cobalamin-binding domains of methionine synthase with methylcobalamin bound. The fold and topology of the amino-terminal helical domain (open bonds) resembles the arrangement of the $E, F, G$ and $H$ helices of the globins, but the corrin is bound on the outside of the helix bundle rather than in its core. The carboxy-terminal $\alpha / \beta$ domain (thin filled bonds) belongs to the general family of nucleotide-binding folds but, as shown in Fig. 5, has evolved features that specifically recognize the lower face and side chain of the cobalamin prosthetic group.

cobalamin and, indeed, no sequence similarities between the methyl- and adenosylcobalamin-dependent proteins have been detected in this vicinity.

In order to accommodate the unique structure of cobalamin, the carboxy-terminal $\alpha / \beta$ domain varies significantly from the canonical nucleotide-binding fold (Rossmann fold). Central elements of this domain surround the dimethylbenzimidazole substituent of the corrin, forming an elongated pocket that accommodates the nucleotide tail (Figs 3 and 4). The third and fourth strands of the parallel $\beta$-sheet line one side of the pocket, which is completed by residues from helices II $\alpha 1$ and II $\alpha 5$. The buried surface area of the side chain car- rying the dimethylbenzimidazole nucleotide accounts for approximately one-half of the total buried area of bound methylcobalamin, suggesting that the interactions of the displaced side chain are important contributors to the overall binding of the cofactor. Comparisons with the nucleotide-binding domain of lactate dehydrogenase (Fig. 5) reveal that helices are not pried away from the sheet to make space for the dimethylbenzimidazole nucleotide. The dimethylbenzimidazole ring system is accommodated partly by the substitution of glycines for the larger side chains of lactate dehydrogenase. In methionine synthase, Gly762, at the start of helix II $\alpha 1$, Gly 802 from strand II $\beta 3$ and Gly 833 from

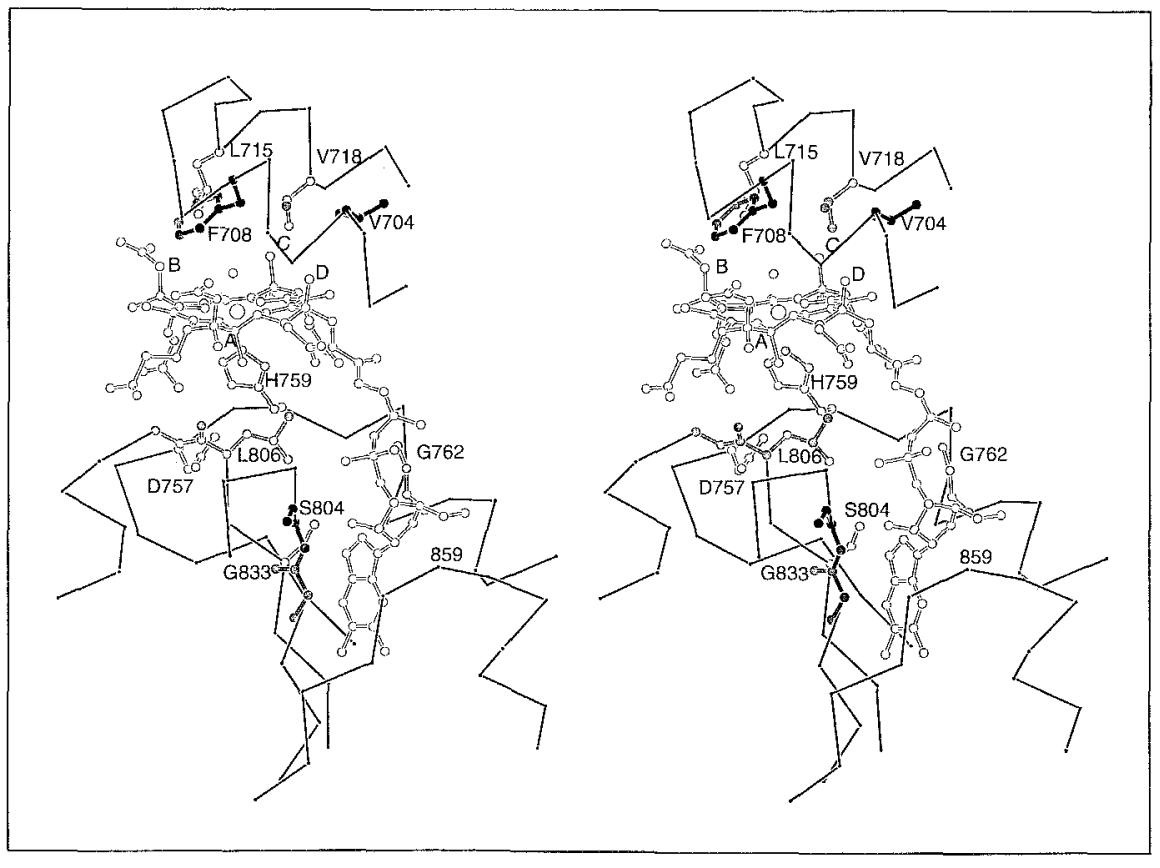

Fig. 4. A close-up view of the methylcobalamin-binding site, rotated $180^{\circ}$ from the vantage point of Fig. 3. The conserved 'fingerprint' residues from the $\alpha / \beta$ domain, drawn in full-atom mode, are below the corrin ring. Hydrophobic residues from the helical domain that contact the upper face of the corrin are also displayed in detail. 
strand II 34 replace the valines found in corresponding sites in lactate dehydrogenase. Truncation of helix II $\alpha 5$ at its amino terminus (near residue 860) opens a site for the ribose- $3^{\prime}$-phosphate moiety of cobalamin. Figure 5 shows the relationship between the binding sites for the dimethylbenzimidazole nucleotide of cobalamin and the nicotinamide riboside of oxidized nicotinamide adenine dinucleotide $\left(\mathrm{NAD}^{+}\right)$. The conformations of the loops at the carboxy-termini of the $\beta$-sheet are another distinquishing feature of the $\alpha / \beta$ domain of methionine synthase. The connecting loop between strand II $\beta 1$ and helix II $\alpha 1$ contributes the His759 ligand to the cobalt instead of binding a nucleotide phosphate. The classic binding site for the adenine nucleotide of pyridine nucleotide-dependent dehydrogenases is obliterated by this loop (Fig. 5). A second loop connecting strand II 33 with helix II $\alpha 3$ is extended at residue 805 and provides hydrophobic contacts with the corrin and polar interactions with propionamide side chains.

\section{A network of hydrogen-bonded residues beneath the corrin may be important in the catalytic reactions of methionine synthase}

A hydrogen-bonded network connects the cobalt and the lower axial ligand, His759, to Ser810 at the surface of the cobalamin-binding fragment and provides a possible pathway for transfer of protons to and from residues near the cobalamin. The $\delta$-nitrogen of the His759 ligand is positioned to form a hydrogen bond to one oxygen of Asp757 (Fig. 4), and Ser810 is oriented to a hydrogen-bond to the other aspartate oxygen. During turnover, methionine synthase must alternately stabilize

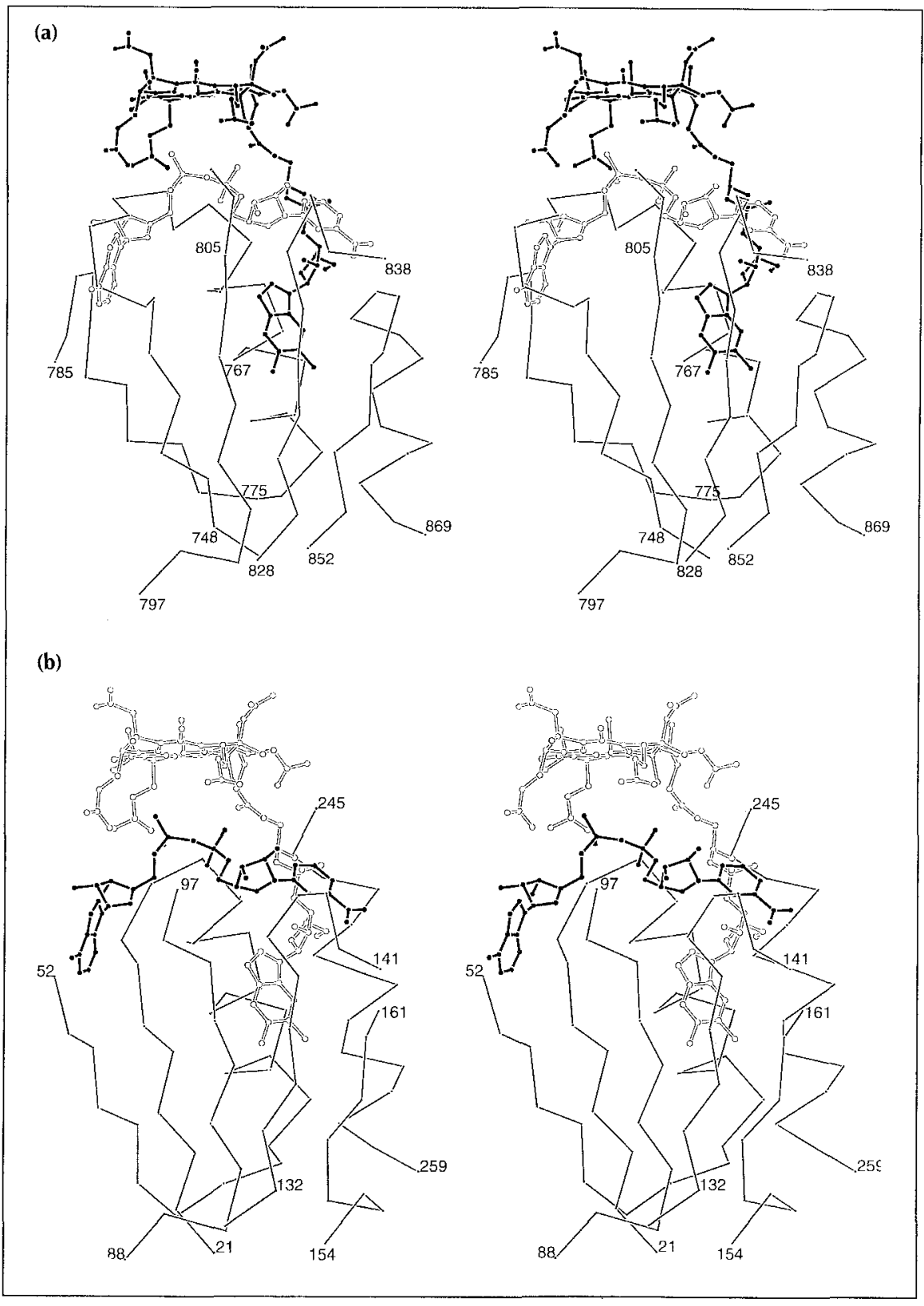

Fig. 5. Comparisons of the folds and cofactor binding of the $\alpha / \beta$ domains of methionine synthase and lactate dehydrogenase [61]. The structures were aligned by matching the backbones of the four central sheet strands. (a) Cobalamin (closed bonds), $\beta$-sheet and other backbone features of methionine synthase, with the oxidized nicotinamide adenine dinucleotide $\left(\mathrm{NAD}^{+}\right)$of lactate dehydrogenase superimposed. (b) Corresponding $\beta$-sheet and bound $\mathrm{NAD}^{+}$of lactate dehydrogenase, with the methylcobalamin of methionine synthase (open bonds) superimposed. Although the corrin macrocycle adjoins the amino-termini of the central sheet strands in methionine synthase, it is significantly displaced from the $\alpha / \beta$ domain, relative to the bound pyridine nucleotide. The adenine nucleotide binding site of lactate dehydrogenase has been replaced by residues $753-760$ and $780-783$ of methionine synthase. Like the nicotinamide nucleotide of lactate dehydrogenase, the dimethylbenzimidazole side chain of the corrin lies on the side of the sheet away from the viewer. However, the sizes and shapes of the binding sites are affected by residue changes (see text), by loops at the ends of strands $\beta 1$ and $\beta 4$, and by the amino terminus of helix $\| \alpha 5$, all of which differ between the two structures. It is clear from these superpositions that the pocket for dimethylbenzimidazole is not equivalent to the classical binding sites for the adenine moieties of pyridine- or flavin-dinucleotides. 
methylcobalamin and cob(I)alamin forms of the prosthetic group (Fig. 1). In model cobalamins, these forms are six- and four-coordinate, respectively. Formation of $\operatorname{cob}(\mathrm{I})$ alamin in methionine synthase is thus assumed to involve dissociation of the His 759 ligand. Protonation and deprotonation of the His759-Asp757 pair, perhaps mediated by Ser810, may play an important role in the conversion between methylcobalamin and cob(I)alamin species [42*0]. We have suggested that the His-Asp pair is deprotonated in the methylcobalamin form of the enzyme, with the histidine partially ionized to the imidazolate form. Protonation of the histidine-aspartate pair accompanying demethylation would favor formation of the cob(I)alamin form of the enzyme. While there is no direct evidence yet for this proposed change in protonation state on going from methylcobalamin to cob(I)alamin, there is direct evidence for the uptake of a proton when the enzyme-bound cofactor is reduced from cob(II)alamin to $\operatorname{cob}(\mathrm{I})$ alamin $\left[38^{\circ}\right]$.

In cytochrome c peroxidase, hydrogen-bonded histidine and aspartate residues are located below the heme iron in an arrangement similar to that seen in methionine synthase In this system as well, reduction of the iron is associated with proton uptake and the redox properties of the heme iron are very sensitive to the precise geometry of the hydrogen-bonding network [44**].

\section{Evidence for structural conservation of the cobalamin-binding $\alpha / \beta$ domain in adenosylcobalamin-dependent enzymes}

Information from the methionine synthase structure and sequence alignments with a subset of six adenosylcobalamin-dependent enzymes (Fig. 6) have been combined to define fingerprint sequences that indicate cobalamin binding. These motifs include many of the conserved residues originally noted by Marsh and co-workers [45]. Although the number of identical residues (bold, Fig. 6) in the alignment is small, many of these invariant residues are in direct contact with cobalamin atoms (Fig. 4) and their conservation can be rationalized from the structure. The paucity of conserved residues may reflect the observation that most of the contacts between the cobalamin side chains and the protein involve either hydrophobic interactions or hydrogen-bonding to backbone atoms rather than to polar side chains of the protein.

The sequences $\mathrm{DxH}^{759} \mathrm{xxG}$ (where $\mathrm{x}$ is any amino acid), SxL 806 and $G^{833}-G^{834}$ seem to be diagnostic for cobalamin binding. The conserved motif $\mathrm{DxH}^{759} \mathrm{xxG}$ forms a loop that passes under the corrin macrocycle, and includes His 759 , the lower axial ligand to the cobalt [42••]. As part of the network for protonation described in the previous section, Asp 757 is hydrogen-bonded to the $\delta$ nitrogen of His 759 , while the side chain of Leu806 contacts the lower face of the corrin and packs against the imidazole ring of His 759 (Fig. 4). The remaining four residues of the fingerprint sequences, Gly762, Ser804 and Gly833-Gly834, interact with the nucleotide tail of the cobalamin (Fig. 4). Gly 762 is located at the beginning of helix II $\alpha 1$, where a side chain would overlap with the phosphate and sugar of the dimethylbenzimidazole nucleotide. The Gly833-Gly834 doublet also appears to be conserved for steric reasons. Side chains in these positions at the carboxyl terminus of IIB4 would block the channel into which dimethylbenzimidazole is inserted. The conserved Ser804 is in position to hydrogen-bond to the $\mathrm{N}^{3}$ of dimethylbenzimidazole in the nucleotide tail.

The conservation of hydrophobic and hydrophilic patches, and the conservation of residues involved in unusual structural features such as a $\beta$ bulge at the carboxyterminal end of strand $I I \beta 2$, predicts that folds closely related to the $\alpha / \beta$ domain of methionine synthase will be found in methylmalonyl CoA mutase and glutamate mutase. The alignments of hydrophobic residues corresponding to the residues in strands II $\beta 1$, IIB3, and II $\beta 4$, which constitute the core of the Rossmann-like domain, are particularly impressive (Fig. 6) The sequence alignments strongly suggest that the fundamental features of the interactions at the lower face of cobalamin, particularly the replacement of dimethylbenzimidazole by a histidine ligand from the protein, will be retained in these adenosylcobalamin-dependent enzymes. In glutamate mutase, the homologous domain is an independently folded subunit of the enzyme $\left[45,46,47^{\circ}\right]$, while in methylmalonyl CoA mutase and methionine synthase this domain is embedded in a larger polypeptide.

The deduced amino acid sequences of some other adenosylcobalamin-dependent enzymes cannot readily be aligned with methionine synthase. However, the conserved sequence $\mathrm{DxH}$ from the cobalamin-dependent ribonucleoside triphosphate reductase from Lactobacillus leichmanii [48*] and from mycobacteriophage L5 [49] may be a fingerprint sequence surrounding a histidyl ligand to adenosylcobalamin, and the large subunits of the ethanolamine ammonia-lyases from Salmonella $t y$ phimurium [50] and Rhodococcus sp. NI86/21 [51] contain the sequences GIEdHfcG and GlEdHfmG, respectively. Blakley et al. [52] reconstituted L. leichmanii ribonucleoside triphosphate reductase with a cobalamin analog containing an adenine nucleotide that assumes a 'base-off' conformation in solution. Electron paramagnetic resonance spectroscopy of the protein reconstituted with the cob(II)alamin analog showed the characteristic nitrogen superhyperfine interactions that indicate coordination of a nitrogen in the lower axial $(\alpha)$ position of the cobalamin. As pointed out by the authors at that time, one interpretation of this finding is that a nitrogenous ligand from the protein becomes coordinated to the cobalt as the base-off analog binds in the active site.

\section{Differential reactivity of protein-bound cobalamins}

Despite the evidence of structural similarity between methionine synthases and adenosylcobalamin-dependent mutases, these two classes of enzymes catalyze heterolytic 


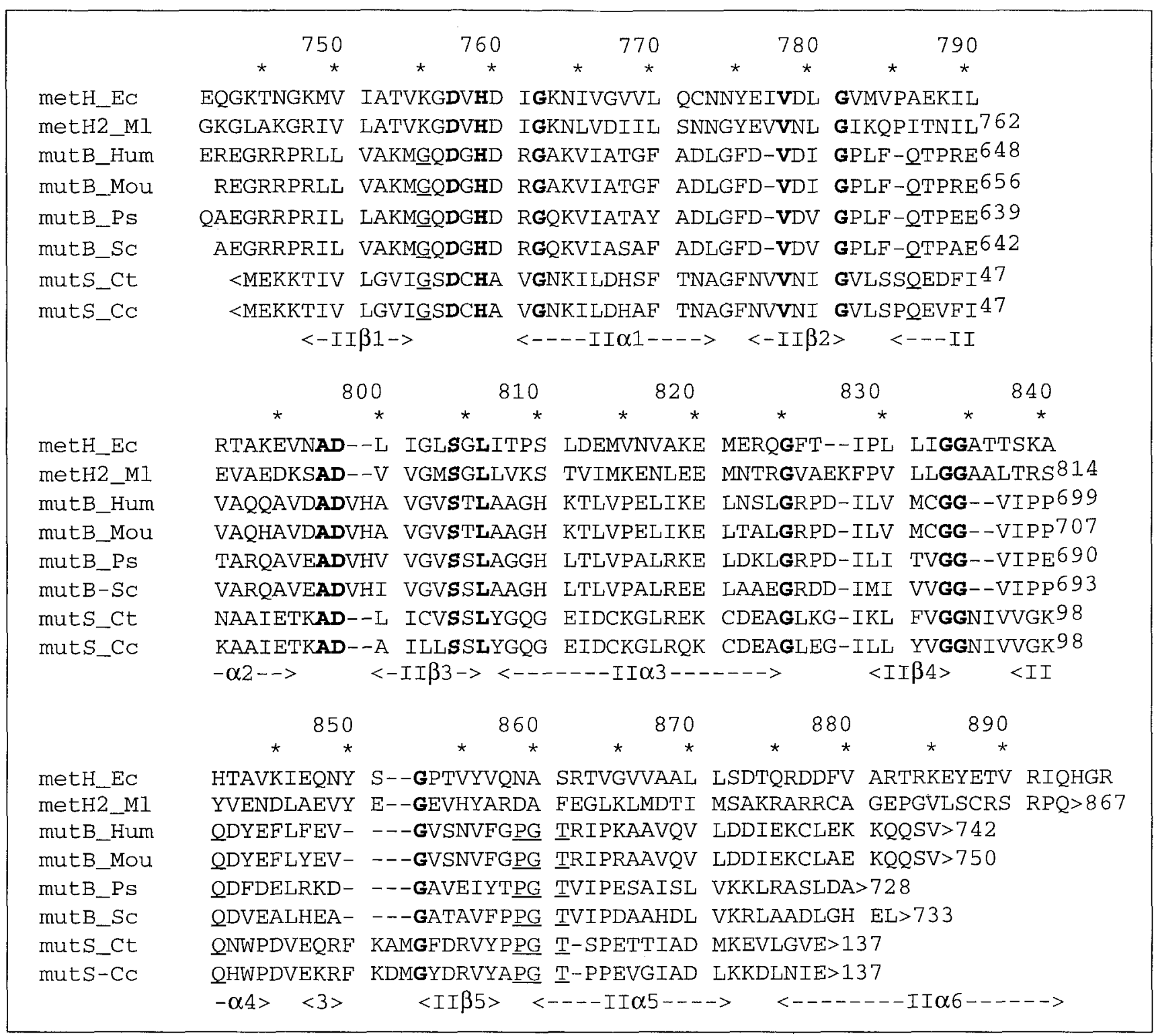

Fig. 6. Alignment of deduced amino acid sequences of a subset of adenosylcobalamin-dependent mutases with homologies to the $\alpha / \beta$ (Rossmann) domain of methionine synthase. Marsh and his co-workers [45] first reported some of the homologies indicated here. The sequences shown are: residues $740-896$ of the deduced amino acid sequence of methionine synthase from Escherichia coli, designated metH-Ec (GenBank Accession \#J04975 [27 ${ }^{\bullet}$ ); residues 712-867 of the deduced amino acid sequence of open reading frame metH 2 from Mycobacterium leprae [40], designated metH2-M1; residues 599-742 of the deduced amino acid sequence obtained from the human cDNA for methylmalonyl-coenzyme $A(\mathrm{COA})$ mutase [62], designated mutB-hum; residues $610-750$ of the deduced amino acid sequence of the mouse methylmalonyl-CoA mutase (GenBank accession \#X57941 [63]), designated mutB-mou; residues 593-728 of the deduced amino acid sequence of the methylmalonyl-CoA mutase MutB subunit from Propionibacterium shermanii (GenBank accession \#X14965 [64]), designated mutB-Ps; residues 596-733 of the deduced amino acid sequence of the methylmalonyl-CoA mutase MutB subunit from Streptomyces cinnamonensis [65]; residues 1-137 comprising the small MutS subunit of glutamate mutase from Clostridium tetanomorphum [45], designated mutS-Ct; and residues 1-137 comprising the deduced amino acid sequence of the glmS gene encoding the small subunit of glutamate mutase from Clostridium cochlearium [46], designated mutS-Cc. Residues shown in bold are conserved in all the aligned sequences; residues that appear to be conserved in adenosylcobalamin-dependent enzymes but not in the methylcobalamin-dependent methyl transferases are underlined. Varations between adenosyl and methyl cobalamin enzymes that could be important include the position of a glycine immediately upstream of the DxHxxG and the appearance of two conserved glutamine residues in all the mutases, corresponding to Ala785 and His841 in MetH. Ser810, a member of the hydrogen-bonded network in methionine synthase, conserved in the methionine synthase from Mycobacterium leprae, is absent from the mutases, which do not form cob(l)alamin during the catalytic cycle.

cleavage of the carbon-cobalt bond on the one hand, and homolytic cleavage on the other. A salient feature of several adenosylcobalamin enzymes is a striking enhancement of the rate of carbon-cobalt bond cleavage on binding of the cofactor to the enzyme-substrate complex [53]. Two basic theories have been proposed to explain the reactivity of cobalamins: a chemical theory holding that the basicity of the lower ligand is the key in modulating the reactivity of the cobalt, and a 'mechanical' theory proposing that deformation of the corrin 
ring system modulates the strength of the carbon-cobalt bond.

The mechanical theory arose from X-ray analyses of a variety of corrins which displayed an 'upward' deformation of the corrin-ring plane $[54,55]$. Repulsive forces between the deformed ring and a bulky upper ligand could weaken the carbon-cobalt bond. The recent Xray structure determination of a vitamin $\mathrm{B}_{12}$ derivative in which imidazole has been substituted for dimethylbenzimidazole in the nucleotide loop [56 $\left.{ }^{\circ}\right]$ shows a decrease in the folding angle of the ring system from $18.0^{\circ}$ to $11.3^{\circ}$, suggesting that the bulky dimethylbenzimidazole is primarily responsible for deformation. For adenosylcobalamin-dependent mutases in which histidine is the lower ligand, it is hard to see how ring flexure can account for increased rates of carbon-cobalt bond cleavage.

Model studies suggest that the ligand in the lower axial position significantly influences the strength of the carbon-cobalt bond in the upper axial position and the propensity for homolytic versus heterolytic cleavage [57-60]. For those adenosylcobalamin enzymes that share the DxH motif with methionine synthase, subtle differences must underlie the different reactivities. As mutational analysis of cytochrome $c$ peroxidase indicates; the reactivity of the metal is highly sensitive to small perturbations in hydrogen bonding of the lower ligand [44**]. Studies of mutant methionine synthases and structural and mutational studies of the mutase enzymes will be crucial for an incisive analysis of the reactivity of protein-bound cobalamins.

\section{Conclusions}

The X-ray structure of the cobalamin-binding region of methionine synthase has revealed unexpected similarities between methylcobalamin-dependent methionine synthase and the adenosylcobalamin-dependent methylmalonyl CoA and glutamate mutases. In particular, it seems very likely that these mutases will bind the cobalamin cofactor with a histidyl residue from the protein displacing the dimethylbenzimidazole nucleotide. The $\alpha / \beta$ domain, a variant of the Rossmann fold, is responsible for stabilizing the dimethylbenzimidazole nucleotide in its displaced position and appears to be structurally conserved in these enzymes. This domain shows significant local divergence from the canonical flavin- or pyridine-nucleotide binding domains. Because of its size, the corrin macrocycle does not enter the crevice that is occupied by nucleotides in the pyridine nucleotide-dependent dehydrogenases. The characteristic site that binds the AMP moiety of $\mathrm{NAD}^{+}$has been lost, and the region that binds the nicotinamide mononucleotide moiety has been reshaped and enlarged to admit the long dimethylbenzimidazole tail. The $\alpha / \beta$ domain also contains residues thought to be responsible for modulating the carbon-cobalt bond strength of the methyl- or adenosylcobalamin cofactor, and its detailed structure is predicted to be extremely important in de- termining whether cleavage of the carbon-cobalt bond is heterolytic or homolytic.

\section{Note added in proof}

Ruma Banerjee and her co-workers [66 $6^{\circ}$ have recently purified methionine synthase from porcine liver to homogeneity, with retention of high activity. They have shown that the porcine enzyme is a monomer of $\sim 155 \mathrm{kDa}$, and that it exhibits an ordered sequential kinetic mechanism similar to that observed for the prokaryotic enzyme.

\section{Acknowledgements}

Research from the authors' laboratories was supported by NIH Grants GM16429 (MLL) and GM 24908 (RGM). CLD was supported by Molecular Biophysics Training Grant GM 08570.

\section{References and recommended reading}

Papers of particular interest, published within the annual period of review, have been highlighted as:

- of special interest

-. of outstanding interest

1. Herbert V, Zalusky $R$ : Interrelations of vitamin $B_{12}$ and folic acid metabolism: folic acid clearance studies. I Clin invest 1962, 41:1263-1276.

2. Noronha $J M$, Silverman $M$ : On folic acid, vitamin $B_{12}$, methionine and formiminoglutamic acid metabolism. In Second european symposium on vitamin $B_{12}$ and intrinsic factor. Edited by Heinrich HC. Stuttgart: Enke; 1962:728-736.

3. Amess JAL, Rees GM, Burman JF, Nancekievill DG, Mollin DL: Megaloblastic haemopoiesis in patients receiving nitrous oxide. Lancet 1978, ii:339-342.

4. Lassen HCA, Henrickson E, Neukirch F, Kristensen HS: Treatment of tetanus: severe bone-marrow depression after prolonged nitrous-oxide anaesthesia. Lancet 1956, i:527-530.

5. Layzer RB: Myeloneuropathy after prolonged exposure to nitrous oxide. Lancet 1978, ii:1227-1230.

6. O'Sullivan $H$, Jennings $F$, Ward $K$, McCann $S$, Scoti JM, Weir $\mathrm{MD}$ : Human bone marrow biochemical function and megaloblastic hematopoiesis after nitrous oxide anesthesia. Anesthesiology 1981, 55:645-649.

7. Banerjee RV, Johnston NL, Sobeski JK, Datta P, Matthews RG: Cloning and sequence analysis of the Escherichia coli meth gene encoding cobalamin-dependent methionine synthase and isolation of a tryptic fragment containing the cobalamitr-binding domain. J Biol Chem 1989, 264:13888-13895.

8. Banerjee RV, Frasca V, Ballou DP, Matthews RG: Participation of cob(1)alamin in the reaction catalyzed by methionine synthase from Escherichia coli: a steady-state and rapid reaction kinetic analysis. Biochemistry 1990, 29:11101-11109.

9. Schrauzer GN, Deutsch E: Reactions of cobalt(I) supernucleophiles. The alkylation of vitamin $B_{12 s}$, cobaloximes (I), and related compounds. J Am Chem Soc 1969, 91:3341-3350.

10. Lexa $D$, Savéant $J-M$ : The electrochemistry of vitamin $\mathbf{B}_{\mathbf{1 2}}$. ACC Chem Res 1983, 16:235-243.

11. Fujii $K$, Huennekens FM: Activation of methionine synthetase by a reduced triphosphopyridine nucleotide-dependent flavoprotein system. I Biol Chem 1974, 249:6745-6753.

12. Drummond JT, Huang S, Blumenthal RM, Matthews RG: As-

- signment of enzymatic function to specific protein regions 
of cobalamin-dependent methionine synthase from Escherichia coli. Biochemistry 1993, 32:9290-9295.

Dissection of methionine synthase by proteolysis and identification of the carboxy-terminal $37 \mathrm{kDa}$ fragment as important for reductive activation of the enzyme by AdoMet.

13. Osborne $C$, Chen L-M, Matthews RG: Isolation, cloning, mapping, and nucleotide sequencing of the gene encoding flavodoxin in Escherichia coli. I Bacteriol 1991, 173:1729-1737.

14. Fujii K, Galivan JH, Huennekens FM: Activation of methionine synthase: further characterization of the flavoprotein system. Arch Biochem Biophys 1977, 178:662-670.

15. Loughlin RE, Elford HL, Buchanan JM: Enzymatic synthesis of the methyl group of methionine. VII. Isolation of a cobalamincontaining transmethylase (5-methyltetrahydrofolate-homocysteine) from mammalian liver. I Biol Chem 1964, 239 2888-2895.

16. Mangum $\mathrm{JH}$, North $\mathrm{JA}$ : Isolation of a cobalamin containing 5-methyltetrahydrofolate-homocysteine transmethylase from mammalian kidney. Biochemistry 1971, 10:3765-3769.

17. Utley CS, Marcell PD, Allen RH, Antony AC, Kolhouse JF: Isolation and characterization of methionine synthase from human placenta. J Biol Chem 1985, 260:13656-13665.

18. Burke $G T$, Mangum JH, Brodie JD: Methylcobalamin as an intermediate in mammalian methionine biosynthesis. Biochemistry 1970, 9:4297-4302.

19. Taylor RT, Weissbach H: Escherichia coli B $N^{5}$-methyltetrahydrofolate-homocysteine methyltransferase: sequential formation of bound methylcobalamin with $S$-adenosyl-I-methionine and N5-methyltetrahydrofolate. Arch Biochem Biophys 1969 129:728-744.

20. Fujii K, Huennekens FM: Methionine synthetase: characterization of protein components and mechanisms for activation and catalysis. In Biochemical aspects of nutrition. Edited by Yagi K. Tokyo: Japan Scientific Societies Press; 1979:173-183.

21. Retey J: Methylmalonyl-CoA mutase. In $B_{12}$. Edited by Dolphin D. New York: John Wiley and Sons; 1982:357-379.

22. Baker JI, Stadtman TC: Amino mutases. In $B_{12}$. Edited by Dolphin D. New York: John Wiley and Sons; 1982:203-232.

23. Switzer R: Glutamate mutase. In $B_{12}$. Edited by Dolphin D. New York: John Wiley and Sons; 1982:289-305.

24. Babior B: Ethanolamine ammonia-lyase. In $B_{12}$. Edited by Dolphin D. New York: John Wiley and Sons; 1982:263-287.

25. Blakley RL: Cobalamin-dependent ribonucleotide reductases. In $B_{12}$. Edited by Dolphin D. New York: John Wiley and Sons; 1982:381-418

26. Old IG, Margarita D, Glass RE, Saint Girons I: Nucleotide sequence of the MetH gene of Escherichia coli K-12 and comparison with that of Salmonella typhimurium LT2. Gene 1990, 87:15-21.

27. Drummond JT, Orgorzalek Loo RR, Matthews RG: Electrospray - mass spectrometric analysis of the domains of a large enzyme: observation of the occupied cobalamin-binding domain and redefinition of the carboxyl terminus of methionine synthase. Biochemistry 1993, 32:9282-9289.

Very useful and novel approaches to resolving discrepancies in sequences deduced from DNA.

28. Luschinsky $\mathrm{CL}$, Drummond JT, Matthews RG, Ludwig ML: Crystallization and preliminary $\mathrm{X}$-ray diffraction studies of the cobalamin-binding domain of methionine synthase from Escherichia coli. / Mol Biol 1992, 225:557-560.

29. Blaschkowski HP, Neuer G, Ludwig-Festl M, Knappe J: Routes of flavodoxin and ferredoxin reduction in Escherichia coli. CoA-acylating pyruvate:flavodoxin and NADPH: flavodoxin oxidoreductases participating in the activation of pyruvate formate-lyase. Eur J Biochem 1982, 123:563-569.

30. Conradt $H$, Hohmann-Berger $M$, Hohmann HP, Blaschkowski HP, Knappe J: Pyruvate formate-lyase (inactive form) and pyruvate formate-lyase activating enzyme of Escherichia coli: iso- lation and structural properties. Arch Biochem Biophys 1984 228:133-142.

31. Eliasson $R$, Fontecave $M$, Jörnvall $H$, Krook $M$, Pontis $E$, Reichard $P$ : The anaerobic ribonucleoside triphosphate reductase from Escherichia coli requires S-adenosylmethionine as a cofactor. Proc Natl Acad Sci USA 1990, 87:3314-3318.

32. Bianchi $V$, Eliasson $R$, Fontecave $M$, Mulliez $E$, Hoover $D M$ - Matthews RG, Reichard P: Flavodoxin is required for the activation of the anaerobic ribonucleotide reductase. Biochem Biophys Res Commun 1993, 197:792-797.

The authors demonstrate a role for $E$. coli flavodoxin in activation of an enzyme by formation of a protein radical.

33. Ifuku O, Kishimoto J, Haze S-I, Yanagi $M$, Fukushima S: Conversion of dethiobiotin to biotin in cell-free extracts of Escherichia coli. Biosci Biotech Biochem 1992, 56:1780-1785.

34. Sanyal I, Cohen G, Flint DH: Biotin synthase: purification,

- characterization as a [2Fe-2S] cluster protein, and in vitro activity of the Escherichia coli bioB gene product. Biochemistry 1994, 33:3625-3631.

AdoMet and an unidentified protein from crude extracts are involved in the activation of purified biotin synthase, extending observations originally made in [33].

35. Frey $M$, Rothe $M$, Wagner AFV, Knappe J: Adenosylmethioninedependent synthesis of the glycyl radical in pyruvate formatelyase by abstraction of the glycine C-2 pro-S hydrogen atom. I Biol Chem 1994, 269:12432-12437.

This study deduces details of the mechanism of activation of pyruvate formate-lyase by AdoMet and flavodoxin in the presence of pyruvate formate-lyase activase.

36. Sun $X$, Harder J, Krook $M$, Jörnvall $H$, Sjöberg B-M, Reichard $P$. - A possible glycine radical in anaerobic ribonucleotide reductase from Escherichia coli: nucleotide sequence of the cloned nrdD gene. Proc Natl Acad Sci USA 1993, 90:577-581.

The sequence of the anaerobic ribonucleotide reductase includes a motif with strong homology to the region containing the glycine backbone radical of active pyruvate-formate lyase, and the authors suggest that the glycine in this motif is oxidized during activation.

37. Mulliez E, Fontcave M Gaillard I, Reichard P: An iron-sulfur center and a free radical in the active anaerobic ribonucleotide reductase of Escherichia coli. I Biol Chem 1993, 268:2296-2299.

Evidence is provided for the generation of an organic free radical on the protein during reductive activation with AdoMet and a reduced nicotinamide adenine dinucleotide phosphate (NADPH)-dependent reducing system.

38. Drummond IT, Matthews RG: Nitrous oxide degradation by cobalamin-dependent methionine synthase: characterization of the reactants and products in the inactivation reaction. Biochemistry 1994, 33:3732-3741.

A group with a redox-linked $\mathrm{pK}$ is identified as taking up a proton on reduction of cob(ll)alamin to cob(l)alamin.

39. Drummond JT, Matthews RG: Nitrous oxide inactivation of

- cobalamin-dependent methionine synthase from Escherichia coli: characterization of the damage to the enzyme and prosthetic group. Biochemistry 1994, 33:3742-3750.

Careful analysis of the reaction of $\mathrm{N}_{2} \mathrm{O}$ with homogenous methionine synthase under controlled conditions provides chemical insights into the in vivo effects of $\mathrm{N}_{2} \mathrm{O}$ on this enzyme.

40. Smith DR: Mycobacterium leprae cosmid B2126. Genbank ACcession \#U00017, 1994

41. Roberts $D L$, Zhao $S$, Doukov $T$, Ragsdale SW: The reduc- tive acetyl-coenzyme A pathway: sequence and heterologous expression of active methyltetra-hydrofolate: corrinoid/ironsulfur protein methyltransferase from Clostridium thermoaceticum. I Bacteriol 1994, 176:6127-6130.

The first 200 amino acids of the sequence of this methyltransferase show homology with the region of the methionine synthase sequence immediately upstream of the cobalamin-binding region.

42. Drennan $\mathrm{CL}$, Huang $\mathrm{S}$, Drummond JT, Matthews RG, Ludwig

*. $M L$ : How a protein binds $B_{12}$ : the $B_{12}$-binding domains of methionine synthase at 3.0A. Science 1994, in press. 
Structure analysis provides the first view of cobalamin bound to a protein. The cofactor changes conformation to allow ligation of His759 to cobalt; the cobalamin is bound by a two domain structure in which the carboxyterminal domain is a variant of the Rossmann nucleotide-binding fold. A hydrogen bonding network involving His759 is proposed to play a role in catalysis.

43. Harris NL, Presnell SR, Cohen FE: Four helix bundle diversity in globular proteins. / Mol Biol 1994, 236:1356-1368.

44. Goodin, DB, McRee, DE: The Asp-His-Fe triad of cytochrome

- $\quad$ c peroxidase controls the reduction potential, electronic structure, and coupling of the trytophan free radical to the heme. Biochemistry 1993, 32:3313-3324.

The authors describe the replacement of aspartate in the triad with glutamine, and a dramatic upward shift in the reduction potential and alteration in its $\mathrm{pH}$-dependence associated with this mutation.

45. Marsh ENG, Holloway DE: Cloning and sequencing of glutamate mutase component $S$ from Clostridium tetanomorphum. FEBS Lett 1992, 310:167-170.

46. Zelder O: C. cochlearium glmS Gene. GenBank/EMBL accession \#X75890, 1994.

47. Holloway DE, Marsh ENG: Adenosylcobalamin-dependent glu- tamate mutase from Clostridium tetanomorphum: overexpresion in Escherichia coli, purification, and characterization of the recombinant enzyme. / Biol Chem 1994, 269:20425-20430.

Independent overexpression in $E$. coli and purification of each of the subunits of glutamate mutase; demonstrated that both large and small subunits are required for cobalamin binding.

48. Booker S, Stubbe J: Cloning, sequencing, and expression of the - adenosylcobalamin-dependent ribonucleotide reductase from Lactobacillus leichmannii. Proc Natl Acad SCi USA 1993, 90:8352-8356

The sequence of $B_{12}$-dependent ribonucleotide reductase has no significant homology with the sequence of the iron/tyrosyl radical-dependent reductase, although in both enzymes cysteines play critical roles.

49. Hatfull GF, Sarkis G]: DNA sequence, structure and gene expression of mycobacteriophage L5: a phage system for mycobacterial genetics. Mol Microbiol 1993, 7:395-405.

50. Faust, LRP, Connor, JA, Roof, DM, Hoch, JA, Babior, BM: Cloning, sequencing and expression of genes encoding the adenosylcobalamin-dependent ethanolamine ammonia-lyase of Salmonella typhimurium. J Biol Chem 1990, 265:12462-12466.

51. De Mot R, Nagy I, Schoofs G, Vanderleyden J: Sequence of a Rhodococcus gene cluster encoding the subunits of ethanolamine ammonia-lyase and an APC-like permease. Gen Bank Accession \#L24492, 1993.

52. Blakley RL, Orme-Johnson WH, Bozdech JM: Mechanism of Lactobacillus leichmannii ribonucleotide reductase: studies with $\operatorname{Co} \alpha-[\alpha$-(aden-9-yl)]-Coß-adenosylcobamide (pseudocoenzyme $B_{12}$ ) as coenzyme. Biochemistry 1979, 18:2335-2339.

53. Finke RG: Coenzyme $B_{12}$-dependent rearrangement reactions: chemical precedent for key elementary steps and insights into enzyme-assisted cobalt-carbon bond cleavage. In Molecular mechanisms in bioorganic processes. Edited by Bleasdale C, Golding BT, Cambridge: Royal Society of Chemistry; 1990:244-280

54. Lenhert PG: The structure of vitamin $B_{12}$. The $X$-ray analysis of the vitamin $B_{12}$ coenzyme. Proc $R$ Soc (London) 1968 A303:45-84.
55. Glusker IP: X-ray crystallography of $\mathbf{B}_{\mathbf{1 2}}$ and cobaloximes. In $B_{12}$. Edited by Dolphin D. New York: John Wiley and Sons; 1982:23-106.

56. Kräutler B, Konrat R, Stupperich E, Färber G, Gruber K, Kratky

- $\quad C$ : Direct evidence for the conformational deformation of the corrin ring by the nucleotide base in vitamin $B_{12}$ : synthesis and solution spectroscopic and crystal structure analysis of $C o \beta$ cyanoimidazolylcobamide. Inorg Chem 1994, 33:4128-4139.

The structure of a model cobalamin in which imidazole rather than dimethylbenzimidazole is the lower $(\alpha)$ ligand to cobalt provides direct evidence for the corrin deformation effected by bulky $\alpha$-ligands.

57. Halpern J: Chemistry and significance of vitamin $\mathbf{B}_{12}$ and model compounds. In $B_{12}$. Edited by Dolphin D. New York: John Wiley and Sons; 1982:501-541.

58. Kräutler $B$ : Thermodynamic trans-effects of the nucleotide base in the $\mathbf{B}_{12}$ coenzymes. Helv Chim Acta 1987, 70:1268-1278.

59. Summers MF, Toscano PJ, Bresciani-Pahor N, Nardin G, Randaccio L, Marzilli LG: A very long cobalt to nitrogen bond in a coenzyme $B_{12}$ model. Relevance to the role of the 5,6-dimethylbenzimidazole in cobalt carbon bond cleavage in coenzyme B ${ }_{12}$. I Am Chem Soc 1983, 105:6259-6263.

60. Summers MF, Marzilli LG, Bresciani-Pahor N, Randaccio L: Unusual bond lengths, conformations, and ligand exchange rates in $B_{12}$ models with the bis(salicylidene)-a-phenylenediamine equatorial ligand. I Am Chem Soc 1984, 106:4478-4485.

61. Rossmann MG, Liljas A, Branden C-I, Banaszak LJ: Evolutionary and structural relationships among dehydrogenases. The Enzymes 1975, Xl:61-102.

62. Jansen $R$, Kalousek F, Fenton WA, Rosenberg LE, Ledley FD: Cloning of full-length methylmalonyl-CoA mutase from a cDNA library using the polymerase chain reaction. Genomics 1989, 4:198-205.

63. Wilkemeyer MF, Crane AM, Ledley FD: Primary structure and activity of mouse methylmaionyl-CoA mutase. Biochem / 1990 271:449-455.

64. Marsh EN, McKie N, Davis NK, Leadlay PF: Cloning and structural characterization of the genes coding for adenosylcobalamin-dependent methylmalonyl-CoA mutase from Propionibacterium shermanii. Biochem, 1989 260:345-352.

65. Birch $A$, Leiser $A$, Robinson JA: Cloning, sequencing, and expression of the gene encoding methylmalonyl-coenzyme $A$ mutase from Streptomyces cinnamonensis. / Bacteriol 1993, 175:3511-3519.

66. Chen Z, Crippen K, Gulati S, Banerjee R: Purification and ki- netic mechanism of a mammalian methionine synthase from the pig liver. I Biol Chem 1994, 269:27193-27197.

This paper reports the purification to homogeneity of the porcine enzyme with retention of high activity. The purified enzyme is a monomer of $-155 \mathrm{kDa}$. It resembles the $B_{12}$-dependent enzyme from $E$. coli in exhibiting an ordered sequential kinetic mechanism in which binding of $\mathrm{CH}_{3}-\mathrm{H}_{4}$ folate precedes binding of homocysteine, and methionine is released before $\mathrm{H}_{4}$ folate.

CL Drennan, RG Matthews and ML Ludwig, Biophysics Re search Division and Department of Biological Chemistry, Chemistry Building, University of Michigan, 930 North University, Ann Arbor, MI 48109-1055, USA. 